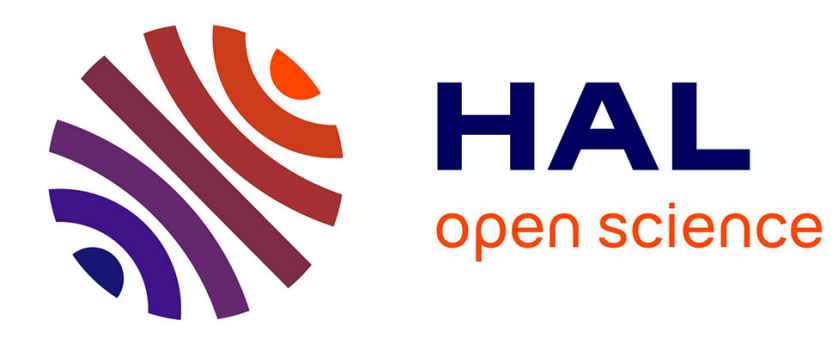

\title{
Some new families of finite elements for the Stokes equations
}

\author{
R. Stenberg
}

\section{To cite this version:}

R. Stenberg. Some new families of finite elements for the Stokes equations. RR-0955, INRIA. 1988. inria-00075604

\section{HAL Id: inria-00075604 https://hal.inria.fr/inria-00075604}

Submitted on 24 May 2006

HAL is a multi-disciplinary open access archive for the deposit and dissemination of scientific research documents, whether they are published or not. The documents may come from teaching and research institutions in France or abroad, or from public or private research centers.
L'archive ouverte pluridisciplinaire HAL, est destinée au dépôt et à la diffusion de documents scientifiques de niveau recherche, publiés ou non, émanant des établissements d'enseignement et de recherche français ou étrangers, des laboratoires publics ou privés. 


\section{Rapports de Recherche}

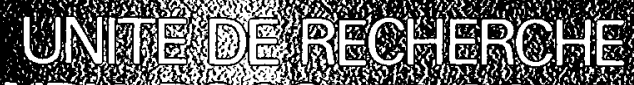

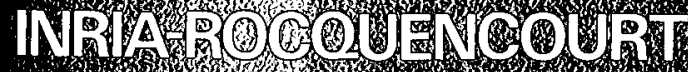

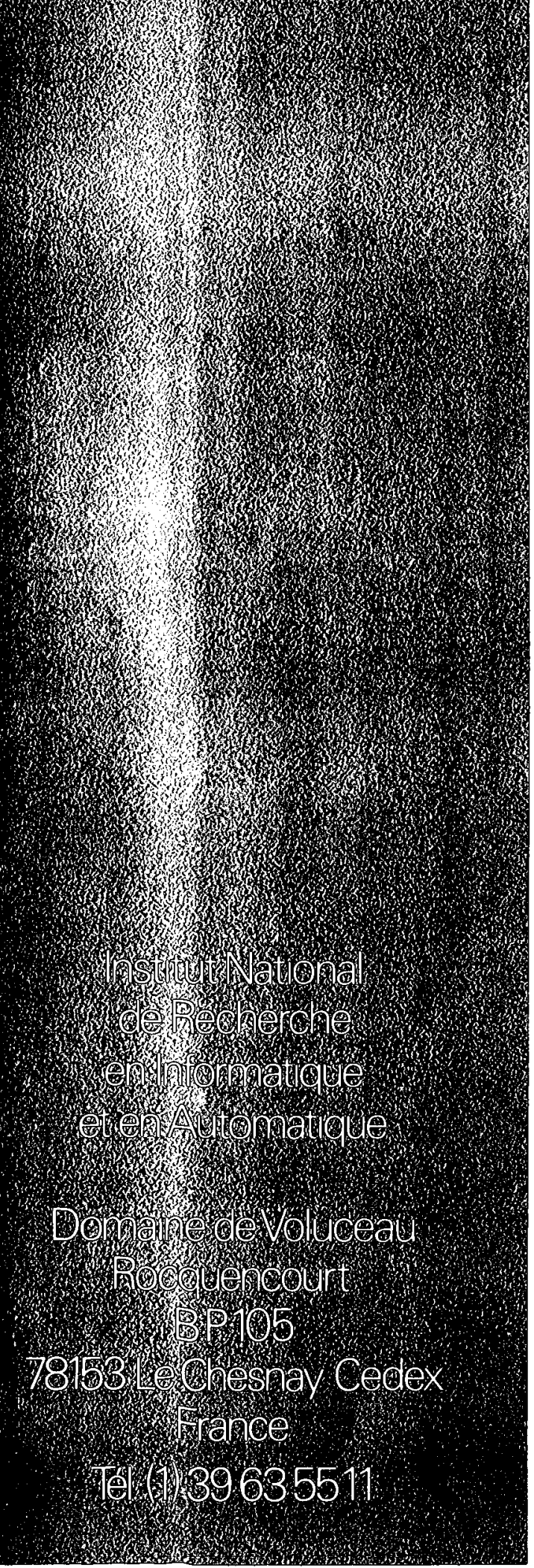




\title{
SOME NEW FAMILIES OF FINITE ELEMENTS FOR THE STOKES EQUATIONS
}

\author{
Rolf Stenberg \\ INRIA Rocquencourt and \\ Institute of Mathematics \\ Helsinki University of Technology \\ 02150 Espoo, Finland
}

\begin{abstract}
We introduce a way of using the mixed finite element families of RaviartThomas-Nedelec [13,14] and Brezzi et al. [5,6,7] for constructing stable and optimally convergent discretizations for the Stokes problem.
\end{abstract}

\section{QUELQUES NOUVELLES FAMILLES D'ELEMENTS FINIS POUR LES EQUATIONS DE STOKES}

Résumé. Nous introduisons une méthode utilisant les familles d'éléments finis mixtes de Raviart-Thomas-Nedelec $[13,14]$ et Brezzi et al. $[5,6,7]$ pour discrétiser le problème de Stokes. Nous montrons la stabilité et la convergence optimale de ces méthodes. 


\section{INTRODUCTION}

Recently there has been introduced a number of new mixed finite element spaces for the approximation of second order elliptic problems $[5,6,7]$. These new methods are of the same type as the methods in the classical Raviart-Thomas-Nedelec families [13,14], but they differ in that they are more accurate for the same computational cost.

One of the reasons for the increased interest in this field is the success of these methods in certain applications. For some problems in geophysics and semiconductor physics they have been shown to be more efficient than more conventional "displacement methods"; cf. $[8,11,19]$ and the references therein.

In this note we will consider the application of these spaces for the approximation of the Stokes equations: Find the velocity $\mathbf{u}$ and the pressure $p$ such that

$$
\begin{aligned}
-\Delta \mathbf{u}+\nabla p=\mathbf{f} & \text { in } \Omega, \\
\operatorname{div} \mathbf{u}=\mathbf{0} & \text { in } \Omega, \\
\mathbf{u}=\mathbf{u}_{0} & \text { on } \partial \Omega,
\end{aligned}
$$

where for simplicity $\Omega \subset \mathbf{R}^{N}, \quad N=2,3$, is assumed to be a bounded polygonal or polyhedral domain.

Let us assume that $\mathbf{f} \in L^{2}(\Omega)^{N}$ and $\mathbf{u}_{0} \in H^{1 / 2}(\partial \Omega)^{N}$ with

$$
\int_{\partial \boldsymbol{\Omega}} \mathbf{u}_{0} \cdot \mathbf{n} d s=0
$$

so that there is a unique solution to (1.1) (as usual the pressure is normalized to have a zero mean value over $\Omega$ ).

When deriving optimal $L^{2}$-estmates for the velocity we will need the usual regularity assumption for the solution to (1.1) when $\mathbf{u}_{0}=0$

$$
\|\mathbf{u}\|_{2}+\|p\|_{1} \leq\|\mathbf{f}\|_{0}
$$


Below we will show that the spaces in $[5,6,7,13,14]$ can be be used as building blocks for producing "Taylor-Hood" type methods for the Stokes problem, i.e. methods with a continuous approximation for the pressure.

Let us here also mention that we in [16] used the same basic finite element spaces for the construction of a family for the discretization of the equations of linear elasticity. The estimates of [16] are uniformly valid with respect to the Poisson ratio, and hence a trivial notational change in the constitutive equation gives yet another family for discretizing Stokes problem.

\section{THE FINITE ELEMENT FAMILIES}

We will give the construction for the triangular and tetrahedral families of $[5,7]$. From the presentation it will be evident that the same construction can be done for the triangular and tetrahedral Raviart-Thomas-Nedelec elements [13,14], and also for all the rectangular and cubic elements of $[5,6,7,13,14]$ except the lowest order methods with a piecewise constant approximation for the velocity. For the rectangular and cubic elements of $[5,6,7]$ all estimates obtained are, however, not optimal.

In order to discretize (1.1) we write it as the system

$$
\begin{aligned}
\sigma-\nabla \mathbf{u}=\mathbf{0} & \text { in } \Omega \\
-\operatorname{div} \sigma+\nabla p=\mathbf{f} & \text { in } \Omega, \\
\operatorname{div} \mathbf{u}=\mathbf{0} & \text { in } \Omega, \\
\mathbf{u}=\mathbf{u}_{\mathbf{0}} & \text { on } \partial \Omega,
\end{aligned}
$$

where $\sigma=\left\{\sigma_{i j}\right\}, \quad \sigma_{i j}=\partial_{j} u_{i}, \quad i, j=1, \ldots, N$, and

$$
(\operatorname{div} \sigma)_{i}=\sum_{j=1}^{N} \partial_{j} \sigma_{i j}, \quad i=1, \ldots, N
$$

(Since no confusion can arise we will use the same notation for the scalar and vector valued divergence operator.) 
Let $\mathcal{C}_{h}$ be the partitioning of $\bar{\Omega}$ into closed triangles or tetrahedrons. The elements of $\mathcal{C}_{h}$ are assumed to be regular in the usual sense (cf. [10]). The quasiuniformity of the mesh will not be assumed.

Our finite element approximation of (2.1) is now defined as: Find $\left(\sigma_{h}, \mathbf{u}_{h}, p_{h}\right) \in H_{h} \times \mathbf{V}_{h} \times$ $P_{h}$ such that

$$
\begin{aligned}
\left(\sigma_{h}, \tau\right)+\left(\operatorname{div} \tau, \mathbf{u}_{h}\right) & =\left\langle\mathbf{u}_{0}, \tau \cdot \mathbf{n}\right\rangle, \quad \tau \in H_{h}, \\
-\left(\operatorname{div} \sigma_{h}, \mathbf{v}\right)+\left(\nabla p_{h}, \mathbf{v}\right) & =(\mathbf{f}, \mathbf{v}), \quad \mathbf{v} \in \mathbf{V}_{h}, \\
\left(\mathbf{u}_{h}, \nabla \boldsymbol{q}\right) & =0, \quad q \in P_{h},
\end{aligned}
$$

where for the index $k \geq 1$ the finite element spaces are defined through

$$
\begin{aligned}
& H_{h}=\left\{\tau \in H(\operatorname{div} ; \Omega) \mid \tau_{\mid K} \in P_{k}(K)^{N \times N}, K \in \mathcal{C}_{h}\right\} \\
& \mathbf{V}_{h}=\left\{\mathbf{v} \in L^{2}(\Omega)^{N} \mid \mathbf{v}_{\mid K} \in P_{k-1}(K)^{N}, K \in \mathcal{C}_{h}\right\} \\
& P_{h}=\left\{p \in C(\Omega) \cap L_{0}^{2}(\Omega) \mid p_{\mid K} \in P_{k}(K), \quad K \in \mathcal{C}_{h}\right\}
\end{aligned}
$$

As usual $(\cdot, \cdot)$ denotes the inner product in $L^{2}(\Omega)^{N}$ or $L^{2}(\Omega)^{N \times N}$, whereas $\langle\cdot, \cdot\rangle$ stands for that in $L^{2}(\partial \Omega)^{N} . L_{0}^{2}(\Omega)$ stands for the subspace of $L^{2}(\Omega)$ consisting of functions with zero mean value over $\Omega$. We point out that for the functions in $H_{h}$ the assumption $\tau \in H(\operatorname{div} ; \Omega)$, where

$$
H(\operatorname{div} ; \Omega)=\left\{\tau \in L^{2}(\Omega)^{N \times N} \mid \operatorname{div} \tau \in L^{2}(\Omega)^{N}\right\}
$$

is equivalent to the continuity of the normal component of $\tau$ along inter element boundaries. We also note that the spaces (2.3a), (2.3b) are $N$ copies of the spaces of Brezzi et al. $[5,7]$.

The finite element method (2.3) gives a poor approximation to the velocity, but it is possible to construct a considerably better approximation by various Element-by-Element Postprocessing Techniques $[1,5,7,17]$. Here we will consider a slight variation of a method introduced by us in [17]. We define

$$
\mathbf{V}_{h}^{*}=\left\{\mathbf{v} \in L^{2}(\Omega)^{N} \mid \mathbf{v}_{\mid K} \in P_{k+1}(K)^{N}, K \in \mathcal{C}_{h}\right\}
$$


and calculate a new approximation $\mathbf{u}_{h}^{*} \in \mathbf{V}_{h}^{*}$ to the velocity separately on each $K \in \mathcal{C}_{h}$ by solving the following problem

$$
\begin{aligned}
\left(\nabla \mathbf{u}_{h}^{*}, \nabla \mathbf{v}\right)_{K} & =\left(\sigma_{h}, \nabla \mathbf{v}\right)_{K}, \quad \mathbf{v} \in\left(I-Q_{h}\right) \mathbf{V}_{h \mid K}^{*}, \\
Q_{h} \mathbf{u}_{h \mid K}^{*} & =\mathbf{u}_{h \mid K},
\end{aligned}
$$

where $Q_{h}: L^{2}(\Omega)^{N} \rightarrow \mathbf{V}_{h}^{*}$ denotes the $L^{2}$-projection and $(\cdot, \cdot)_{K}$ stands for the inner product in $L^{2}(K)^{N \times N}$.

REMARK. We note that the postprocessing is performed separately for each component of $\mathbf{u}$ and that the local stiffness matrices to invert on each element are the same for all components.

The analysis technique to be utilized in this paper is the one based on mesh dependent norms, first introduced in [2] for certain mixed approximations of the biharmonic equation and later adapted for mixed methods for second order problems [15]. The norms to be used are the following

$$
\begin{aligned}
& \|\sigma\|_{0, h}^{2}=\|\sigma\|_{0}^{2}+\sum_{T \in \Gamma_{h}} h_{T} \int_{T}|\sigma \cdot \mathbf{n}|^{2} d s, \\
& \quad \text { for } \sigma \in L^{2}(\Omega)^{N \times N} \text { with } \sigma \cdot \mathbf{n} \in L^{2}(T)^{N}, T \in \Gamma_{h}, \\
& \left.\|\mathbf{u}\|_{1, h}^{2}=\sum_{K \in \mathcal{C}_{h}}\|\nabla \mathbf{u}\|_{0, K}^{2}+\sum_{T \in \Gamma_{h}} h_{T}^{-1} \int_{T} \| \mathbf{u}\right]\left.\right|^{2} d s \\
& \quad \text { for } \mathbf{u} \in L^{2}(\Omega)^{N} \text { with } \mathbf{u}_{\mid K} \in H^{1}(K)^{N}, \quad K \in \mathcal{C}_{h},
\end{aligned}
$$

and

$$
\|\sigma, \mathbf{u}\|_{h}^{2}=\|\sigma\|_{0, h}^{2}+\|\mathbf{u}\|_{1, h}^{2}
$$

Here $T$ stands for a side of an element, $\mathbf{n}$ is the normal to $T$ and $\Gamma_{h}$ denotes the collection of the sides of the elements of $\mathcal{C}_{h}$. For element sides in the interior of $\Omega[\mathbf{u}]$ denotes the jump in $\mathbf{u}$ whereas for sides on $\partial \Omega$ it denotes the value of $\mathbf{u}$. We note that an integration by part on each $K \in \mathcal{C}_{h}$ yields

$$
(\operatorname{div} \sigma, \mathbf{u}) \leq\|\sigma\|_{0, h}\|\mathbf{u}\|_{1, h}
$$


for each $\sigma$ and $\mathbf{u}$

ments (cf. [2]) gives the interpolation estimates

$$
\inf _{\tau \in H_{h}}\|\sigma-\tau\|_{0, h} \leq C h^{r}|\sigma|_{r}, \quad 1 / 2<r \leq k+1
$$

and

$$
\inf _{\mathbf{v} \in \mathrm{V}_{h}}\|\mathbf{u}-\mathbf{v}\|_{1, h} \leq C h^{s-1}|\mathbf{u}|_{s}, \quad 1 \leq s \leq k
$$

In order to see that the problem (2.2) can be analyzed with Brezzi's theory of saddle-point problems [3] we write it in the following way: Find $\left(\sigma_{h}, \mathbf{u}_{h}, p_{h}\right) \in H_{h} \times \mathbf{V}_{h} \times P_{h}$ such that

$$
\begin{gathered}
a\left(\sigma_{h}, \mathbf{u}_{h} ; \tau, \mathbf{v}\right)+b\left(\tau, \mathbf{v} ; p_{h}\right)=(\mathbf{f}, \mathbf{v})+\left\langle\mathbf{u}_{0}, \tau \cdot \mathbf{n}\right\rangle, \quad(\tau, \mathbf{v}) \in H_{h} \times \mathbf{V}_{h} \\
b\left(\sigma_{h}, \mathbf{u}_{h} ; q\right)=0, \quad q \in P_{h},
\end{gathered}
$$

where

$$
a(\sigma, \mathbf{u} ; \tau, \mathbf{v})=(\sigma, \tau)+(\operatorname{div} \tau, \mathbf{u})-(\operatorname{div} \sigma, \mathbf{v})
$$

and

$$
b(\tau, \mathbf{v} ; p)=(\mathbf{v}, \nabla p)
$$

Hence, the analysis now consist of the verification of the two following lemmas.

LEMMA 2.1. There is a positive constant $C$ such that

$$
\sup _{\substack{o \neq(\sigma, \mathbf{u}) \epsilon \\ H_{h} \times \mathbf{V}_{h}}} \frac{a(\sigma, \mathbf{u} ; \tau, \mathbf{v})}{\|\sigma, \mathbf{u}\|_{h}} \geq C\|\tau, \mathbf{v}\|_{h} \quad(\tau, \mathbf{v}) \in H_{h} \times \mathbf{V}_{h}
$$

PROOF: This inequality states the stability of the discretization of the (vector) Laplace operator by the finite element spaces (2.3). This is a well known fact $[5,7]$ even if it is usually not stated in this form. Let us therefore sketch the proof.

By applying the theory of Brezzi [3] we note that the assertion follows from the the following stability estimate

$$
\sup _{\substack{\tau \in H_{h} \\\|\tau\|_{0, h}=1}}(\operatorname{div} \tau, \mathbf{u}) \geq C\|\mathbf{u}\|_{1, h} \quad \mathbf{u} \in \mathrm{V}_{h}
$$


To prove this it is sufficient to take $\tau$ in the Raviart-Thomas-Nedelec subspace $\tilde{H}_{h} \subset H_{h}$, defined through

$\tilde{H}_{h}=\left\{\sigma \in H(\operatorname{div} ; \Omega) \mid\left(\sigma_{i 1}, \ldots, \sigma_{i N}\right)_{\mid K} \in P_{k-1}(K)^{N} \oplus \tilde{P}_{k-1}(K) \mathbf{x}, K \in \mathcal{C}_{h}, i=1, \ldots, N\right\}$

where $\tilde{P}_{k-1}(K)$ is the space of homogeneous polynomials of degree $k-1$. In $[13,14]$ it is shown that for the degrees of freedom for $\tau \in \tilde{H}_{h}$ one can choose

$$
\begin{aligned}
& \text { (i) }\langle\tau \cdot \mathbf{n}, \mathbf{z}\rangle_{T}, \quad \mathbf{z} \in P_{k}(T)^{N}, T \subset \partial K, \\
& \text { (ii) } \quad(\tau, \nabla \mathbf{w})_{K}, \quad \mathbf{w} \in P_{k-1}(K)^{N},
\end{aligned}
$$

for each $K \in \mathcal{C}_{h}$.

Now an integration by parts on each $K \in \mathcal{C}_{h}$ yields

$$
(\operatorname{div} \tau, \mathbf{u})=\sum_{K \in \mathcal{C}_{h}}-(\tau, \nabla \mathbf{u})_{K}+\sum_{T \in \Gamma_{h}}\langle\tau \cdot \mathbf{n},[\mathbf{u}]\rangle_{T}
$$

Hence, since $([\mathbf{u}])_{\mid T} \in P_{k}(T)^{N}, \quad T \in \Gamma_{h}$ and $\mathbf{u} \in P_{k-1}(K)^{N}$ for $K \in \mathcal{C}_{h}$, we can choose $\tau \in \tilde{H}_{h}$ such that

$$
\begin{array}{lll}
\text { (i) } \quad \tau \cdot \mathbf{n}_{\mid T}=h_{T}^{-1 / 2}([\mathbf{u}])_{\mid T}, & T \in \Gamma_{h}, \\
\text { (ii) } \quad(\tau, \nabla \mathbf{u})_{K}=\|\nabla \mathbf{u}\|_{0, K}^{2}, & K \in \mathcal{C}_{h} .
\end{array}
$$

This gives

$$
(\operatorname{div} \tau, \mathbf{u})=\|\mathbf{u}\|_{1, h}^{2}
$$

By scaling (cf. $[2,14])$ one proves that

$$
\|\tau\|_{0, h} \leq C\|\mathbf{u}\|_{1, h}
$$

which verifies the assertion.

LEMMA 2.2. There is a positive constant $C$ such that

$$
\sup _{\substack{o \neq(\sigma, \mathbf{u}) \in \in \\ H_{h} \times \mathbf{v}_{h}}} \frac{b(\sigma, \mathbf{u} ; q)}{\|\sigma, \mathbf{u}\|_{h}} \geq C\|q\|_{0} \quad q \in P_{h}
$$


PROOF: Since for $q \in P_{h}$ it holds $\nabla q \in \mathbf{V}_{h}$, we can choose $\mathbf{u}$ through

$$
\mathbf{u}_{\mid K}=h_{K}^{2} \nabla q_{\mid K}, \quad K \in \mathcal{C}_{h}
$$

With $\sigma=0$ this gives

$$
b(\sigma, \mathbf{u} ; q)=\sum_{K \in \mathcal{C}_{h}} h_{K}^{2}\|\nabla q\|_{0, K}^{2}
$$

Scaling arguments yield

$$
\|\mathbf{u}\|_{1, h}^{2} \leq C \sum_{K \in \mathcal{C}_{h}} h_{K}^{-2}\|\mathbf{u}\|_{0, K}^{2}=C \sum_{K \in \mathcal{C}_{h}} h_{K}^{2}\|\nabla q\|_{0, K}^{2}
$$

By combining (2.12) and (2.13) we get

$$
\sup _{\substack{\sigma \neq(\sigma, u) \in \in \\ B_{h} \times V_{h}}} \frac{b(\sigma, \mathbf{u} ; q)}{\|\sigma, \mathbf{u}\|_{h}} \geq C\left(\sum_{K \in \mathcal{C}_{h}} h_{K}^{2}\|\nabla q\|_{0, K}^{2}\right)^{1 / 2}
$$

The assertion now follows from an argument by Verfürth [18, Proposition 3.3]. We remark that by using the above locally weighted norm for the pressure, the quasiuniformity assuption (i.e. $h_{K} \geq C h \forall K \in \mathcal{C}_{h}$ ) of [18] can be avoided.

We now get the following error estimates.

THEOREM 2.1. For the solution $\mathbf{u}_{h}^{*} \in \mathrm{V}_{h}^{*}$ and $p_{h} \in P_{h}$ to (2.5) and (2.2) we have

$$
\left\|\mathbf{u}-\mathbf{u}_{h}^{*}\right\|_{1, h}+\left\|p-p_{h}\right\|_{0} \leq C h^{k+1}\left(|\mathbf{u}|_{k+2}+|p|_{k+1}\right) .
$$

Moreover, if (1.2) is valid we have

$$
\begin{gathered}
\left\|\mathbf{u}-\mathbf{u}_{h}^{*}\right\|_{0} \leq C h^{k+2}\left(|\mathbf{u}|_{k+2}+|p|_{k+1}\right) \quad \text { for } k \geq 2, \\
\left\|\mathbf{u}-\mathbf{u}_{h}^{*}\right\|_{0} \leq C h^{2}\left(|\mathbf{u}|_{3}+|p|_{2}\right) \quad \text { for } \quad k=1
\end{gathered}
$$

If in addition $\mathbf{f} \in \mathbf{V}_{h}$, then (2.14) is also valid for $k=1$.

PROOF: Let us split the proof into four steps. 
Step 1. Let us prove the estimate

$$
\left\|\sigma-\sigma_{h}\right\|_{0, h}+\left\|\mathbf{u}_{h}-Q_{h} \mathbf{u}\right\|_{1, h}+\left\|p-p_{h}\right\|_{0} \leq C h^{k+1}\left(|\sigma|_{k+1}+|p|_{k+1}\right)
$$

Lemmas 2.1 and 2.2 implies the existence of a triple $(\tau, \mathbf{v}, q) \in H_{h} \times \mathrm{V}_{h} \times P_{h}$ such that

$$
\|\tau\|_{0, h}+\|\mathbf{v}\|_{1, h}+\|q\| \leq C
$$

and

$$
\begin{aligned}
& \left\|\sigma_{h}-\tilde{\sigma}\right\|_{0, h}+\left\|\mathbf{u}_{h}-Q_{h} \mathbf{u}\right\|_{1, h}+\left\|p_{h}-\tilde{p}\right\| \\
& \leq a\left(\sigma_{h}-\tilde{\sigma}, \mathbf{u}_{h}-Q_{h} \mathbf{u} ; \tau, \mathbf{v}\right)+b\left(\tau, \mathbf{v} ; p_{h}-\tilde{p}\right)+b\left(\sigma_{h}-\tilde{\sigma}, \mathbf{u}_{h}-Q_{h} \mathbf{u} ; q\right)
\end{aligned}
$$

where $\tilde{\sigma}$ and $\tilde{p}$ are the interpolants to $\sigma$ and $p$, respectively. Using (2.1) and (2.2) we get

$$
\begin{aligned}
& a\left(\sigma_{h}-\tilde{\sigma}, \mathbf{u}_{h}-Q_{h} \mathbf{u} ; \tau, \mathbf{v}\right)+b\left(\tau, \mathbf{v} ; p_{h}-\tilde{p}\right)+b\left(\sigma_{h}-\tilde{\sigma}, \mathbf{u}_{h}-Q_{h} \mathbf{u} ; q\right) \\
& =a\left(\sigma-\tilde{\sigma}, \mathbf{u}-Q_{h} \mathbf{u} ; \tau, \mathbf{v}\right)+b(\tau, \mathbf{v} ; p-\tilde{p})+b\left(\sigma-\tilde{\sigma}, \mathbf{u}-Q_{h} \mathbf{u} ; q\right) \\
& =(\sigma-\tilde{\sigma}, \tau)+\left(\mathbf{u}-Q_{h} \mathbf{u}, \operatorname{div} \tau\right)-(\mathbf{v}, \operatorname{div}(\sigma-\tilde{\sigma})) \\
& +(\mathbf{v}, \nabla(p-\tilde{p}))+\left(\mathbf{u}-Q_{h} \mathbf{u}, \nabla q\right) .
\end{aligned}
$$

Let us now estimate the terms on the right hand side of (2.18). Since $\nabla q \in V_{h}$ and $\operatorname{div} \tau \in \mathrm{V}_{h}$ the definition of $Q_{h}$ yields

$$
\left(\mathbf{u}-Q_{h} \mathbf{u}, \operatorname{div} \tau\right)=0
$$

and

$$
\left(\mathbf{u}-Q_{h} \mathbf{u}, \nabla q\right)=0
$$

Further, standard interpolation theory gives

$$
(\sigma-\tilde{\sigma}, \tau) \leq\|\sigma-\tilde{\sigma}\|_{0}\|\tau\|_{0} \leq C h^{k+1}|\sigma|_{k+1},
$$

and using (2.8) and (2.9) we get

$$
(\mathbf{v}, \operatorname{div}(\sigma-\tilde{\sigma})) \leq C\|\sigma-\tilde{\sigma}\|_{0, h}\|\mathbf{v}\|_{1, h} \leq C h^{k+1}|\sigma|_{k+1}
$$


Finally, the last term is estimated as follows

$$
\begin{aligned}
& (\mathbf{v}, \nabla(p-\tilde{p}))=-\sum_{K \in \mathcal{C}_{h}}(\operatorname{div} \mathbf{v}, p-\tilde{p})+\sum_{T \in \Gamma_{h}} \int_{T}([\mathbf{v} \cdot \mathbf{n}])(p-\tilde{p}) d s \\
& \left.\leq\left.\left(\sum_{K \in \mathcal{C}_{h}}\|\operatorname{div} \mathbf{v}\|_{0, K}^{2}+\sum_{T \in \Gamma_{h}} h_{T}^{-1} \int_{T} \| \mathbf{v} \cdot \mathbf{n}\right]\right|^{2} d s\right)^{1 / 2} \cdot\left(\|p-\tilde{p}\|_{0}^{2}+\sum_{T \in \Gamma_{h}} h_{T} \int_{T}|p-\tilde{p}|^{2} d s\right)^{1 / 2} \\
& \leq C\|\mathbf{v}\|_{1, h}\left(\|p-\tilde{p}\|_{0}^{2}+\sum_{T \in \Gamma_{h}} h_{T} \int_{T}|p-\tilde{p}|^{2} d s\right)^{1 / 2} \\
& \leq C h^{k+1}|p|_{k+1},
\end{aligned}
$$

where we in the last step used an interpolation estimate easily obtained by scaling; cf. [2,Lemma 3].

By collecting (2.17)-(2.23), using the triangle inequality and (2.9) we get (2.15) which contains the asserted estimate for the pressure.

Step 2. Let us derive an estimate for $\left\|Q_{h}\left(\mathbf{u}_{h}^{*}-\mathbf{u}\right)\right\|_{0}$.

We first note that $Q_{h} \mathbf{u}_{h}^{*}=\mathbf{u}_{h}$.

Suppose that (1.2) is valid so that the solution $(\mathbf{z}, \gamma, q) \in H_{0}^{1}(\Omega)^{N} \times L^{2}(\Omega)^{N \times N} \times L_{0}^{2}(\Omega)$ to

$$
\begin{aligned}
\gamma-\nabla \mathbf{z} & =0 & & \text { in } \Omega, \\
-\operatorname{div} \gamma+\nabla q & =\mathbf{u}_{h}-Q_{h} \mathbf{u} & & \text { in } \Omega, \\
\operatorname{div} \mathbf{z} & =0 & & \text { in } \Omega, \\
\mathbf{z} & =0 & & \text { on } \partial \Omega,
\end{aligned}
$$

satisfies

$$
\|\mathbf{z}\|_{2}+\|\gamma\|_{1}+\|q\|_{1} \leq\left\|\mathbf{u}_{h}-Q_{h} \mathbf{u}\right\|_{0}
$$

Let $\tilde{\gamma}$ and $\tilde{q}$ be the interpolants to $\gamma$ and $q$. Using the relations

$$
(\operatorname{div} \tilde{\gamma}, \mathbf{u})=\left(\operatorname{div} \tilde{\gamma}, Q_{h} \mathbf{u}\right) \text { and }(\nabla \tilde{q}, \mathbf{u})=\left(\nabla \tilde{q}, Q_{h} \mathbf{u}\right)
$$


standard arguments gives

$$
\begin{aligned}
& \left\|\mathbf{u}_{h}-Q_{h} \mathbf{u}\right\|_{0}^{2} \\
& =\left(\sigma-\sigma_{h}, \tilde{\gamma}-\gamma\right)+\left(\operatorname{div}\left(\sigma-\sigma_{h}\right), \mathbf{z}-Q_{h} \mathbf{z}\right)-\left(\nabla\left(p-p_{h}\right), \mathbf{z}-Q_{h} \mathbf{z}\right) \\
& +\left(\operatorname{div} \gamma, Q_{h} \mathbf{u}-\mathbf{u}_{h}\right)-\left(\operatorname{div} \tilde{\gamma}, \mathbf{u}-\mathbf{u}_{h}\right)-\left(\nabla q, Q_{h} \mathbf{u}-\mathbf{u}_{h}\right)+\left(\nabla \tilde{q}, \mathbf{u}-\mathbf{u}_{h}\right) \\
& =\left(\sigma-\sigma_{h}, \tilde{\gamma}-\gamma\right)+\left(\operatorname{div}\left(\sigma-\sigma_{h}\right), \mathbf{z}-Q_{h} \mathbf{z}\right)-\left(\nabla\left(p-p_{h}\right), \mathbf{z}-Q_{h} \mathbf{z}\right) \\
& +\left(\operatorname{div}(\gamma-\tilde{\gamma}), Q_{h} \mathbf{u}-\mathbf{u}_{h}\right)-\left(\nabla(q-\tilde{q}), Q_{h} \mathbf{u}-\mathbf{u}_{h}\right) \\
& \leq C\left(\left\|\sigma-\sigma_{h}\right\|_{0, h}+\left\|\mathbf{u}_{h}-Q_{h} \mathbf{u}\right\|_{1, h}+\left\|p-p_{h}\right\|_{0}+\left(\sum_{T \in \Gamma_{h}} h_{T} \int_{T}\left|p-p_{h}\right|^{2} d s\right)^{1 / 2}\right) \\
& \cdot\left(\|\gamma-\tilde{\gamma}\|_{0, h}+\left\|\mathbf{z}-Q_{h} \mathbf{z}\right\|_{1, h}+\|q-\tilde{q}\|_{0}+\left(\sum_{T \in \Gamma_{h}} h_{T} \int_{T}|q-\tilde{q}|^{2} d s\right)^{1 / 2}\right) \\
& \leq C h^{k+1}\left(|\sigma|_{k+1}+|p|_{k+1}\right) \\
& \cdot\left(\|\gamma-\tilde{\gamma}\|_{0, h}+\left\|\mathbf{z}-Q_{h} \mathbf{z}\right\|_{1, h}+\|q-\tilde{q}\|_{0}+\left(\sum_{T \in \Gamma_{h}} h_{T} \int_{T}|q-\tilde{q}|^{2} d s\right)^{1 / 2}\right) .
\end{aligned}
$$

For $k \geq 2,(2.9),(2.10)$ and the interpolation estimate of [2, Lemma 3] give

$$
\begin{aligned}
& \left(\|\gamma-\tilde{\gamma}\|_{0, h}+\left\|\mathbf{z}-Q_{h} \mathbf{z}\right\|_{1, h}+\|q-\tilde{q}\|_{0}+\left(\sum_{T \in \Gamma_{h}} h_{T} \int_{T}|q-\tilde{q}|^{2} d s\right)^{1 / 2}\right) \\
& \leq C h\left(|\gamma|_{1}+|q|_{1}+|\mathbf{z}|_{2}\right) .
\end{aligned}
$$

Recalling that $Q_{h} \mathbf{u}_{h}^{*}=\mathbf{u}_{h},(2.25)-(2.27)$ give

$$
\left\|Q_{h}\left(\mathbf{u}_{h}^{*}-\mathbf{u}\right)\right\|_{0} \leq C h^{k+2}\left(|\sigma|_{k+1}+|p|_{k+1}\right) \quad \text { for } k \geq 2 \text {. }
$$

For the case $k=1$ and $\mathbf{f} \in \mathrm{V}_{h}$ we have

$$
\begin{aligned}
& \left(\operatorname{div}\left(\sigma-\sigma_{h}\right)+\nabla\left(p-p_{h}\right), \mathbf{z}-Q_{h} \mathbf{z}\right) \\
& =\left(\mathbf{f}-\operatorname{div} \sigma_{h}+\nabla p_{h}, \mathbf{z}-Q_{h} \mathbf{z}\right)=0
\end{aligned}
$$

and (2.28) is still valid.

If $\mathbf{f} \notin \mathbf{V}_{h}$ we only get

$$
\left\|Q_{h}\left(\mathbf{u}_{h}^{*}-\mathbf{u}\right)\right\|_{0} \leq C h^{2}\left(|\sigma|_{2}+|p|_{2}\right) \quad \text { for } k=1
$$


Step 3. Next we estimate $\left\|\left(I-Q_{h}\right)\left(\mathbf{u}_{h}^{*}-\mathbf{u}\right)\right\|_{0}$ and $\left\|\left(I-Q_{h}\right)\left(\mathbf{u}_{h}^{*}-\mathbf{u}\right)\right\|_{1, h}$.

First, since $\left(I-Q_{h \mid K}\right) \mathbf{v}_{\mid K}=0$ for $\mathbf{v} \in P_{0}(K)^{N}$, we have

$$
\left\|\left(I-Q_{h}\right)\left(\mathbf{u}_{h}^{*}-\mathbf{u}\right)\right\|_{0, K} \leq C h_{K}\left|\left(I-Q_{h}\right)\left(\mathbf{u}_{h}^{*}-\mathbf{u}\right)\right|_{1, K}
$$

Next we write

$$
\begin{aligned}
& \left|\left(I-Q_{h}\right)\left(\mathbf{u}_{h}^{*}-\mathbf{u}\right)\right|_{1, K}^{2}=\left(\nabla\left(I-Q_{h}\right)\left(\mathbf{u}_{h}^{*}-\mathbf{u}\right), \nabla\left(I-Q_{h}\right)\left(\mathbf{u}_{h}^{*}-\mathbf{u}\right)\right)_{K} \\
& =\left(\nabla\left(\mathbf{u}_{h}^{*}-\mathbf{u}\right), \nabla\left(I-Q_{h}\right)\left(\mathbf{u}_{h}^{*}-\mathbf{u}\right)\right)_{K}-\left(\nabla Q_{h}\left(\mathbf{u}_{h}^{*}-\mathbf{u}\right), \nabla\left(I-Q_{h}\right)\left(\mathbf{u}_{h}^{*}-\mathbf{u}\right)\right)_{K}
\end{aligned}
$$

Now, (2.1) and (2.5) gives

$$
\begin{aligned}
& \left(\nabla\left(\mathbf{u}_{h}^{*}-\mathbf{u}\right), \nabla\left(I-Q_{h}\right)\left(\mathbf{u}_{h}^{*}-\mathbf{u}\right)\right)_{K}=\left(\sigma_{h}-\sigma, \nabla\left(I-Q_{h}\right)\left(\mathbf{u}_{h}^{*}-\mathbf{u}\right)\right)_{K} \\
& \leq\left\|\sigma-\sigma_{h}\right\|_{0, K}\left|\left(I-Q_{h}\right)\left(\mathbf{u}_{h}^{*}-\mathbf{u}\right)\right|_{1, K}
\end{aligned}
$$

Further, a local inverse inequality yields

$$
\begin{aligned}
& \left(\nabla Q_{h}\left(\mathbf{u}_{h}^{*}-\mathbf{u}\right), \nabla\left(I-Q_{h}\right)\left(\mathbf{u}_{h}^{*}-\mathbf{u}\right)\right)_{K} \\
& \left.\leq\left|Q_{h}\left(\mathbf{u}_{h}^{*}-\mathbf{u}\right)\right|_{1, K} \mid\left(I-Q_{h}\right)\left(\mathbf{u}_{h}^{*}-\mathbf{u}\right)\right)\left.\right|_{1, K} \\
& \left.\leq C h_{K}^{-1}\left\|Q_{h}\left(\mathbf{u}_{h}^{*}-\mathbf{u}\right)\right\|_{0, K} \mid\left(I-Q_{h}\right)\left(\mathbf{u}_{h}^{*}-\mathbf{u}\right)\right)\left.\right|_{1, K}
\end{aligned}
$$

Combining (2.30)-(2.33) and summing over all $K \in \mathcal{C}_{h}$ gives

$$
\left\|\left(I-Q_{h}\right)\left(\mathbf{u}_{h}^{*}-\mathbf{u}\right)\right\|_{0} \leq C\left(h\left\|\sigma-\sigma_{h}\right\|_{0}+\left\|Q_{h}\left(\mathbf{u}_{h}^{*}-\mathbf{u}\right)\right\|_{0}\right)
$$

Also, from $(2.31),(2.32),(2.33)$ and the inequality (proven by scaling)

$$
h_{K}^{-1} \int_{\partial K}\left|\left(I-Q_{h}\right) \mathbf{v}\right|^{2} d s \leq C\left|\nabla\left(I-Q_{h}\right) \mathbf{v}\right|_{0, K}^{2}, \quad \mathbf{v} \in \mathbf{V}_{h \mid K}^{*}
$$

we get

$$
\left\|\left(I-Q_{h}\right)\left(\mathbf{u}_{h}^{*}-\mathbf{u}\right)\right\|_{1, h} \leq C\left(\left\|\sigma-\sigma_{h}\right\|_{0}+\left\|Q_{h}\left(\mathbf{u}_{h}^{*}-\mathbf{u}\right)\right\|_{1, h}\right)
$$

Step 4. By combining $(2.28),(2.29),(2.34)$ and (2.15) we obtain the asserted estimates for $\left\|\mathbf{u}-\mathbf{u}_{h}^{*}\right\|_{0}$, and that for $\left\|\mathbf{u}-\mathbf{u}_{h}^{*}\right\|_{1, h}$ follows from (2.15) and (2.35). 


\section{The implementation by hybrydization}

Let us close the paper by briefly considering the solution of the discrete system (2.2). The method advocated in $[1,5,6,7]$ is the Fraijs de Veubeke hybridization technique. In that the condition $\operatorname{div} \sigma \in L^{2}(\Omega)^{N}$ for $\sigma \in H_{h}$, which is equivalent to the continuity of $\sigma \cdot \mathbf{n}$ along interelement boundaries, is enforced by introducing Lagrange multipliers. More precisely, define

$$
\mathbf{M}_{h}=\left\{\mathbf{m} \mid \mathbf{m}_{\mid T} \in P_{k}(T)^{N}, T \in \Gamma_{h}, \mathbf{m}_{\mid T}=0 \text { for } T \subset \partial \Omega\right\},
$$

and let $\hat{H}_{h}$ be as in (2.3a) with the condition $\operatorname{div} \sigma \in L^{2}(\Omega)^{N}$ dropped. The modified discretization now reads: Find $\left(\sigma_{h}, \mathbf{u}_{h}, \mathbf{m}_{h}, p_{h}\right) \in \hat{H}_{h} \times \mathbf{V}_{h} \times \mathbf{M}_{h} \times P_{h}$ such that

$$
\begin{aligned}
\left(\sigma_{h}, \tau\right)+\sum_{K \in \mathcal{C}_{h}}\left\{\left(\operatorname{div} \tau, \mathbf{u}_{h}\right)_{K}-\left\langle\tau \cdot \mathbf{n}_{K}, \mathbf{m}_{h}\right\rangle_{\partial K}\right\} & =\left\langle\tau \cdot \mathbf{n}, \mathbf{u}_{0}\right\rangle, \quad \tau \in \hat{H}_{h}, \\
-\sum_{K \in \mathcal{C}_{h}}\left(\operatorname{div} \sigma_{h}, \mathbf{v}\right)_{K}+\left(\nabla p_{h}, \mathbf{u}\right) & =(\mathbf{f}, \mathbf{v}), \quad \mathbf{v} \in \mathbf{V}_{h}, \\
\left(\mathbf{u}_{h}, \nabla q\right) & =0, \quad q \in P_{h}, \\
\sum_{K \in \mathcal{C}_{h}}\left\langle\sigma_{h} \cdot \mathbf{n}_{K}, \mathbf{l}\right\rangle_{\partial K} & =0, \quad l \in \mathbf{M}_{h} .
\end{aligned}
$$

Above $\mathbf{n}_{K}$ stands for the unit outward normal to $\partial K$.

Clearly, the three first components of (2.2) and (3.1) coincide. The physical meaning of the new unknown $\mathbf{m}_{\boldsymbol{h}}$ is that of an approximation to the velocity $\mathbf{u}$ along the inter element boundaries.

For $\mathbf{m}_{h}$ we have the following error estimate which is needed if any of the postprocessing methods of $[1,5,7]$ is used.

LEMMA 4.1. For $T \in \Gamma_{h}$ let $I_{T}: L^{2}(T)^{N} \rightarrow \mathbf{M}_{h \mid T}$ be the $L^{2}$-projection. Then we have

$$
\left\|\mathbf{m}_{h}-I_{T} \mathbf{u}\right\|_{0, T} \leq C\left\{h_{K}^{1 / 2}\left\|\sigma-\sigma_{h}\right\|_{0, K}+h_{K}^{-1 / 2}\left\|Q_{h} \mathbf{u}-\mathbf{u}_{h}\right\|_{0, K}\right\}
$$

for $T \subset \partial K, K \in \mathcal{C}_{h}$.

For the proof of this result we refer to [7, Lemma 4.1]. 
The algebraic equations generated by (3.1) are of the form

$$
\begin{aligned}
\mathcal{A} \Sigma+\mathcal{B U}-\mathcal{C} \mathrm{M} & =0 \\
-\mathcal{B}^{T} \Sigma+\mathcal{D} P & =\mathrm{F} \\
\mathcal{C}^{T} \Sigma & =0 \\
\mathcal{D}^{T} \mathrm{U} & =0
\end{aligned}
$$

where $\Sigma, \mathrm{U}, \mathrm{M}$ and $\mathrm{P}$ are the vectors for the degrees of freedom for $\sigma_{h}, \mathbf{u}_{h}, \mathbf{m}_{h}$ and $p_{h}$, respectively.

Now the matrix $\mathcal{A}$ is positively definite and block diagonal. Hence, $\Sigma$ can be eliminated separately on each element which gives the system

$$
\begin{aligned}
\hat{\mathcal{A}} \hat{\mathrm{U}}+\hat{\mathcal{D}} \mathrm{P} & =\hat{\mathrm{F}}, \\
\hat{\mathcal{D}}^{T} \hat{\mathrm{U}} & =0
\end{aligned}
$$

where $\hat{U}^{T}=(\mathrm{U}, \mathrm{M})^{T}, \hat{\mathcal{D}}^{T}=\left(\mathcal{D}^{T}, \quad 0\right), \hat{\mathrm{F}}^{T}=(\mathrm{F}, \quad 0)^{T}$ and

$$
\hat{\mathcal{A}}=\left(\begin{array}{cc}
\mathcal{B}^{T} \mathcal{A}^{-1} \mathcal{B}^{T} & -\mathcal{B}^{T} \mathcal{A}^{-1} \mathcal{C} \\
-\mathcal{C}^{T} \mathcal{A}^{-1} \mathcal{B} & \mathcal{C}^{T} \mathcal{A}^{-1} \mathcal{C}
\end{array}\right)
$$

which is positively definite. Hence the system is of the same form as for more conventional Taylor-Hood type discretizations of Stokes problem. Thus any of the methods for solving linear systems of this kind (cf. [12]) can be utilized.

Further, $\mathcal{B}^{T} \mathcal{A}^{-1} \mathcal{B}^{T}$ is also positively definite and block diagonal, and hence $\mathrm{M}$ can be eliminated on each element saparately. This results in a system of the form

$$
\begin{aligned}
\mathcal{E} \mathrm{M}+\mathcal{G P} & =\mathrm{G}, \\
\mathcal{G}^{T} \mathrm{M}-\mathcal{H P} & =\mathrm{H},
\end{aligned}
$$

where both $\mathcal{E}$ and $\mathcal{H}$ are symmetric and positively definite. Now the system has the same form as that of some recent stabilized mixed methods $[4,9]$. 


\section{REFERENCES}

[1] D.N. ARNOLD and F. BREZZI, Mixed and Nonconforming Finite Element Methods: Implementation, Postprocessing and Error Estimates, RAIRO. M $^{2}$ AN, Vol. 19, 1985, pp. 7-32.

[2] I. BABUŠKA, J.E. OSBORN and J. PITKÄRANTA, Analysis of Mixed Methods Using Mesh Dependent Norms, Math. Comp., Vol. 35, 1980, pp. 1039-1062.

[3] F. BREZZI, On the Existence, Uniqueness and Approximation of Saddle-point Problems arising from Lagrange Multipliers, RAIRO Ser. Rouge Vol. 8, 1974, pp. 129-151.

[4] F. BREZZI and J. DOUGLAS, Stabilized Mixed Methods for Stokes Problem, Numer. Math., Vol. 53, 1988, pp. 225-236.

[5] F. BREZZI, J. DOUGLAS, R. DURÁN and M. FORTIN, Mixed Finite Elements for Second Order Elliptic Problems in Three Variables, Numer. Math., Vol. 51, 1987, pp. 237-250.

[6] F. BREZZI, J. DOUGLAS, M. FORTIN and L.D. MARINI, Efficient Rectangular Mixed Finite Elements in Two and Three Space Variables, RAIRO. $\mathrm{M}^{2} \mathrm{AN}$, Vol. 21, 1987, pp. 181-204

[7] F. BREZZI, J. DOUGLAS and L.D. MARINI, Two Families of Mixed Finite Elements for Second Order Elliptic Equations, Numer. Math., Vol. 47, 1985, pp. 19-34.

[8] F. BREZZI, L.D. MARINI and P. PIETRA, Two-Dimensional Exponential Fitting and Applications to Semiconductor Device Equations, Preprint

[9] F. BREZZI and J. PITKÄRANTA, On the Stabilization of Finite Element Approximations for the Stokes Problem, Notes on Numerical Fluid Mechanics Vol. 10. W. Hackbusch (Ed.), Vieweg, Braunschweig 1984, pp. 11-19

[10] P.G. CIARLET, The Finite Element Method for Elliptic Problems, North - Holland 1978. 
[11] J. DOUGLAS and J.E. SANTOS, Approximation of Waves in Composite Media, The Mathematics of Finite Elements and Applications VI. MAFELAP 1987, J. R. Whiteman (Ed.), Acamemic Press, London 1988, pp. 55-74

[12] V. GIRAULT and P.A. RAVIART, Finite Element Methods for Navier-Stokes Equations. Theory and Algorithms, Springer 1986.

[13] J.C. NEDELEC, Mixed Finite Elements in $\mathbf{R}^{3}$, Numer. Math., Vol. 35, 1980, pp. 315-341.

[14] P.A. RAVIART and J.M. THOMAS, A Mixed Finite Element Method for 2nd Order Elliptic Problems, Proceedings of the Symposium on the Mathematical Aspects of the Finite Element Method. Lecture Notes in Mathematics 606, Springer 1977, pp. 292-315.

[15] R. STENBERG, On the Construction of Optimal Mixed Finite Element Methods for the Linear Elasticity Problem, Numer. Math., Vol. 48, 1986, pp. 447-462.

[16] R. STENBERG, A Family of Mixed Finite Elements for the Elasticity Problem, Numer. Math. 1988, To appear

[17] R. STENBERG, Postprocessing Schemes for Some Mixed Finite Elements, Rapport de recherche No. 800, INRIA Fevrier 1988

[18] R. VERFÜRTH, Error Estimates for a Mixed Finite Element Approximation of the Stokes Equations, RAIRO Anal. Numer. Vol. 18, 1984, pp. 175-182

[19] M. WHEELER and R. GONZALES, Mixed Finite Element Methods for Petroleum Reservoir Simulation, Computing Methods in Applied Sciences and Engineering, VI, R. Glowinski, J.L. Lions (Eds.), North-Holland 1984, pp. 639-658. 
. 